\title{
Genetic Mapping of Social Interaction Behavior in B6/MSM Consomic Mouse Strains
}

\author{
Aki Takahashi $\cdot$ Kazuya Tomihara $\cdot$ \\ Toshihiko Shiroishi · Tsuyoshi Koide
}

Received: 30 June 2009/ Accepted: 2 November 2009/Published online: 20 November 2009

(c) The Author(s) 2009. This article is published with open access at Springerlink.com

\begin{abstract}
Genetic studies are indispensable for understanding the mechanisms by which individuals develop differences in social behavior. We report genetic mapping of social interaction behavior using inter-subspecific consomic strains established from MSM/Ms (MSM) and C57BL/6J (B6) mice. Two animals of the same strain and sex, aged 10 weeks, were introduced into a novel openfield for $10 \mathrm{~min}$. Social contact was detected by an automated system when the distance between the centers of the two animals became less than $\sim 12 \mathrm{~cm}$. In addition, detailed behavioral observations were made of the
\end{abstract}

Edited by Stephen Maxson.

Electronic supplementary material The online version of this article (doi:10.1007/s10519-009-9312-x) contains supplementary material, which is available to authorized users.

A. Takahashi $\cdot$ T. Koide $(\bowtie)$

Mouse Genomics Resource Laboratory, National Institute of Genetics, 1111 Yata, Mishima, Shizuoka 411-8540, Japan e-mail: tkoide@lab.nig.ac.jp

\section{K. Tomihara}

Department of Psychology, Faculty of Law, Economics and Humanities, Kagoshima University, Kohrimoto, Kagoshima 890-0065, Japan

\section{T. Shiroishi}

Mammalian Genetics Laboratory, National Institute of Genetics, 1111 Yata, Mishima, Shizuoka 411-8540, Japan

A. Takahashi - T. Shiroishi - T. Koide

Department of Genetics, SOKENDAI, Hayama,

Kanagawa 240-0193, Japan

Present Address:

A. Takahashi

Department of Psychology, Tufts University, 530 Boston

Avenue (Bacon Hall), Medford, MA 02155, USA males. The wild-derived mouse strain MSM showed significantly longer social contact as compared to B6. Analysis of the consomic panel identified two chromosomes (Chr 6 and $\mathrm{Chr}$ 17) with quantitative trait loci (QTL) responsible for lengthened social contact in MSM mice and two chromosomes ( $\mathrm{Chr} 9$ and $\mathrm{Chr} \mathrm{X}$ ) with QTL that inhibited social contact. Detailed behavioral analysis of males identified four additional chromosomes associated with social interaction behavior. B6 mice that contained Chr 13 from MSM showed more genital grooming and following than the parental B6 strain, whereas the presence of Chr 8 and Chr 12 from MSM resulted in a reduction of those behaviors. Longer social sniffing was observed in $\mathrm{Chr} 4$ consomic strain than in B6 mice. Although the frequency was low, aggressive behavior was observed in a few pairs from consomic strains for Chrs 4, 13, 15 and 17, as well as from MSM. The social interaction test has been used as a model to measure anxiety, but genetic correlation analysis suggested that social interaction involves different aspects of anxiety than are measured by open-field test.

Keywords Social interaction behavior .

Consomic mouse strains (CSS) · Wild-derived MSM .

Quantitative trait loci $\cdot$ Anxiety $\cdot$ Aggression

\section{Introduction}

In addition to environmental and epigenetic effects, individual differences in social behavior result from genetic influences. The analysis of transgenic and knockout mice has enabled the successful identification of genes associated with a number of types of social behavior, including social affiliation, social recognition, sexual behavior, 
maternal care, and aggressive behavior (Choleris et al. 2004; Pfaff et al. 2002; Gammie 2005; Donaldson and Young 2008; Nelson and Chiavegatto 2001; Miczek et al. 2001). In humans, a missense mutation in the monoamine oxidase A (MAOA) gene drastically changes social behavior in males (Brunner et al. 1993a, b). However, in general, it is more likely that natural diversity in social behavior arises from multigenic influences that result from the additive or interactive effects of alleles that occur naturally, rather than the lack or over-expression of a single gene product. To understand this behavioral diversity within species, forward genetics approaches such as quantitative trait loci (QTL) analysis have been used (Flint 2003). However, a limited number of QTL studies on social behavior have been performed; only two studies have been reported that identify genetic loci associated with mouse aggressive behavior (Brodkin et al. 2002; Roubertoux et al. 2005). QTL studies for social behavior are limited because of the amount of time and effort that is required to assess social behavior: QTL studies require the analysis of at least several hundred animals to achieve statistical significance.

The other issue that affects QTL analysis of social behavior is the heterogeneity of the animals analyzed. In social interaction tests using inbred strains, the social behaviors observed are the result of interactions between animals of the same genotype. However, to perform QTL analysis of social behavior, it is necessary to use a heterogenic population and to consider the interaction of two different genotypes. To perform such QTL analysis, a common opponent from a particular strain is introduced to an F2 or an N2 individual. However, the social behavior is influenced by the genotype of both the test subject and the opponent (Fuller and Hahn 1976). Indeed, it has been reported that aggressive behavior can change depending on the genotype of the opponent (Selmanoff et al. 1976; Maxson and Canastar 2003; Ogawa et al. 2004; Roubertoux et al. 2005). Therefore, a method that allows QTL analysis of social behavior between animals of the same genotype would be very beneficial.

Consomic strains, which are also known as chromosome substitution strains (CSSs), should provide a useful resource for the genetic mapping of social interaction behavior. Consomic strains have the same genetic background with the exception that, in each strain, one chromosome has been replaced with the corresponding chromosome from a different inbred strain (Nadeau et al. 2000). Therefore, it is possible to use consomic strains to examine social behavior between individuals of the same genotype.

In this study, we used a panel of B6-ChrN ${ }^{\mathrm{MSM}}$ consomic mouse strains, which were established from the wildderived mouse strain MSM/Ms (MSM) and the laboratory strain C57BL/6J (B6; Takada et al. 2008). MSM was derived from Japanese wild mice (Mus musculus molossinus) and inbred at the National Institute of Genetics (NIG; Mishima, Japan) since 1978 (Moriwaki et al. 2009). Although they have been inbred in the laboratory for more than 60 generations, they still retain the characteristics of wildness to a great extent (Koide et al. 2000; Takahashi et al. 2006). Previously, we have investigated anxiety-like behavior, using a panel of B6-ChrN ${ }^{\mathrm{MSM}}$ consomic mice by the open-field test, light/dark test, and elevated plus-maze test, and successfully mapped loci that are related to anxiety-like behavior (Takahashi et al. 2008). In the present study, we characterized social interaction behavior between MSM and B6 mice in a novel open-field, and then we identified chromosomes that were involved in social interaction behavior, using a panel of $\mathrm{B} 6-\mathrm{ChrN}^{\mathrm{MSM}}$ consomic strains. The social interaction test has been used to measure anxiety and is sensitive to the administration of anxiolytic drugs (File and Hyde 1978; File 1980). However, it has been suggested that social interaction behavior has a different genetic basis to other anxiety-like behavior (Sano et al. 2009; O'Tuathaigh et al. 2008). Therefore, we investigated social interaction behavior in detail and its genetic correlation with other conventional anxiety-like behavior.

\section{Materials and methods}

\section{Animals}

MSM was established as an inbred strain after 20 generations of brother-sister mating at the NIG (Moriwaki et al. 2009). B6 mice were purchased from CLEA Japan (Tokyo, Japan) and bred at the NIG. Development of the B6-ChrN ${ }^{\mathrm{MSM}}$ consomic panel has been described in detail previously (Takada et al. 2008). All consomic strains had the same genetic background as B6, except for one pair of chromosomes, which were replaced with the corresponding chromosomes from MSM. It proved difficult to substitute the whole chromosome for $\mathrm{Chr}$ 2, Chr 6, Chr 7 and $\mathrm{Chr} 12$. Therefore, two subconsomic strains that carried the telomeric (T) and centromeric (C) portions, respectively, and that covered the whole chromosome were established for each of these chromosomes. The consomic strains for $\mathrm{Chr}$ 5, Chr 7C and Chr 10 did not breed well, and the behavior of these strains could not be characterized. Each consomic strain was described as $\mathrm{B} 6-\mathrm{ChrN}^{\mathrm{MSM}}$, in which $\mathrm{N}$ was the number of the chromosome transferred from the MSM strain. In total, 517 pairs of male or female mice were tested: 28 pairs of B6, 20 pairs of MSM, and 469 pairs of consomic mice (Table 1). The animals were 10 weeks old at the time of testing. All animals were maintained at the 
Table 1 Numbers of pairs tested in this study

\begin{tabular}{|c|c|c|c|}
\hline & & Male & Female \\
\hline \multicolumn{2}{|l|}{ B6 } & 14 & 14 \\
\hline \multicolumn{2}{|l|}{ MSM } & 10 & 10 \\
\hline \multirow[t]{22}{*}{ Consomic mouse strains } & 1 & 13 & 11 \\
\hline & $2 \mathrm{C}$ & 10 & 11 \\
\hline & $2 \mathrm{~T}$ & 11 & 11 \\
\hline & 3 & 11 & 11 \\
\hline & 4 & 11 & 11 \\
\hline & $6 \mathrm{C}$ & 10 & 11 \\
\hline & $6 \mathrm{~T}$ & 12 & 11 \\
\hline & $7 \mathrm{~T}$ & 13 & 11 \\
\hline & 8 & 11 & 10 \\
\hline & 9 & 11 & 10 \\
\hline & 11 & 10 & 10 \\
\hline & $12 \mathrm{C}$ & 11 & 11 \\
\hline & $12 \mathrm{~T}$ & 11 & 10 \\
\hline & 13 & 11 & 10 \\
\hline & 14 & 11 & 11 \\
\hline & 15 & 16 & 12 \\
\hline & 16 & 11 & 11 \\
\hline & 17 & 11 & 11 \\
\hline & 19 & 12 & 10 \\
\hline & $\mathrm{XC}$ & 10 & 10 \\
\hline & XT & 10 & 7 \\
\hline & $Y$ & 11 & - \\
\hline
\end{tabular}

NIG under a 12/12 h light/dark cycle (light from 08:00 to $20: 00 \mathrm{~h})$ in a temperature-controlled room $\left(23 \pm 2^{\circ} \mathrm{C}\right)$. The mice were weaned around 3-4 weeks of age and housed in same-sex groups in standard-sized plastic cages on wood chips, until the behavioral tests were performed. All tests were carried out from 16:00 to 20:00 h. Food and water were available ad libitum. Mice were maintained according to NIG guidelines, and all procedures were approved by the Institutional Committee for Animal Care and Use.

\section{Social interaction test}

The open-field used consisted of a square arena $(60 \times 60 \times 40 \mathrm{~cm})$ made of a white polyvinylchloride plastic board and lit by incandescent lighting (80 lux). Two littermates of the same sex were used for each test, after having been kept as cage-mates. Before starting the behavioral tests, each mouse was kept in isolation for 10 days (de Angelis and File 1979). The two same-sex mice were placed gently in opposite corners of the field, and allowed to move freely for $10 \mathrm{~min}$. To analyze the total number of occurrences of social contact, and their duration, the movement of the mice in the arena was recorded continuously by a video camera mounted over its center. The signal was relayed to a video tracking system (Image SI; O'Hara \& Co., Tokyo, Japan), which was based on a public domain image processing and analysis program, NIH Image, from the National Institute of Health (USA). Social contact was recognized when the centers of the two mice came within a distance of $\sim 12 \mathrm{~cm}$ (equivalent to 20 pixels). In addition to video tracking analysis, we performed detailed behavioral observations. The duration and number of occurrences of the following five types of behavior were recorded from the video images: social sniffing (sniffing several body parts of the other mouse, excluding the anogenital region); genital grooming (sniffing the anogenital region of the other mouse); following (following behind the other mouse while touching the nose to the other's body); attack (which included biting, lunging, wrestling, and offensive lateral attack); and aggressive chasing (chasing the other animal immediately after attack). Ten or more pairs were examined for each strain.

\section{Statistics}

First, we performed two-way ANOVA to examine the effect of strain and sex on the duration and frequency of social contact for B6 and MSM. For genital grooming, social sniffing, and following behavior, we analyzed only males, and therefore, we performed one-way ANOVA to determine the effect of strain. Next, two-way ANOVA with strain (B6 and all consomic strains) and sex was performed. The significance of each consomic strain compared to B6 was determined by a $t$-test with Bonferroni correction as post-hoc tests. As a comparison, $Z$ scores were also estimated by using Eq. (3) from Belknap (2003), which is a standard method used to examine the effect of consomic strains (Supplemental Table). Several types of behavior (social sniffing, genital grooming, and following) did not show a normal distribution, and therefore, were subjected to logarithmic conversion $[\log (x+1)]$ prior to statistical analysis. Duration of attack and aggressive chasing were categorized together as aggressive behavior. As a result of the low number of pairs that showed aggressive behavior, we did not perform statistical analysis on this variable.

Pearson's correlations among social interaction behavior types were calculated by using the mean score for each consomic strain and for B6 to estimate genetic correlations (Blizard and Bailey 1979). The data that were used to calculate the genetic correlations were from the male mice because we performed detailed analysis only on males. To estimate whether genetic loci affect both males and females similarly or either sex independently, Pearson's correlations between means of males and females were calculated for duration and frequency of social contact. We also 
calculated Pearson's correlations between social interaction and anxiety-like behavior. For anxiety-like behavior, we used data from our previously published paper (Takahashi et al. 2008), and factor scores for males of each strain were used to estimate the genetic correlations.

\section{Results}

Social interaction behavior in B6 and MSM mice

Two-way ANOVA showed a significant main effect of strain on the duration of social contact $[F(1,44)=$ 130.632, $P<0.001]$ : social contact in MSM mice was $\sim 3$ times longer than that in B6 mice for both sexes (Fig. 1a). There was no main effect or interaction with sex. With respect to frequency of social interaction, there was a significant interaction between strain and sex $[F(1,44)=$ 4.553, $P=0.038$ ) but no main effect of strain or sex was observed. Male MSM mice showed a higher frequency of social contact than female mice, whereas the opposite was observed in B6 mice; however, these differences were shown to be not significant by the post-hoc test (Fig. 1a).
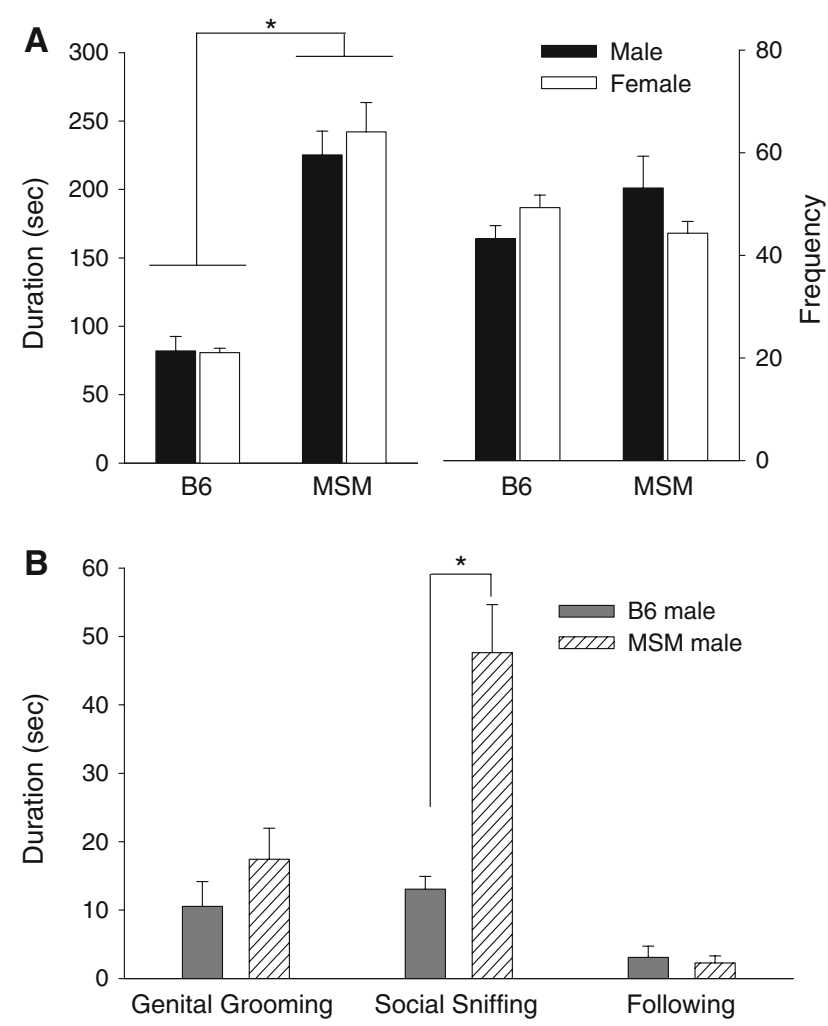

Fig. 1 Social interaction behavior in B6 and MSM mice. a Duration (left side) and frequency (right side) of social contact. b Duration of detailed social interaction behavior (genital grooming, social sniffing, and following). Bars represent mean \pm SEM. Asterisk indicates significant difference between B6 and MSM $(P<0.001)$
Agonistic behaviors were observed in some male pairs, but not in pairs of female mice, thus we performed detailed behavioral observations for the male mice using video recording. Detailed analysis revealed that the longer duration of social contact in MSM mice that was detected by the automatic video tracking analysis resulted from higher levels of social sniffing, as compared to the B6 mice $[F(1,23)=27.893, P<0.001)$. There was no significant difference in genital grooming or following behavior between the two strains (Fig. 1b). Aggressive behavior, including aggressive chasing and attack biting, was observed in three out of 11 pairs of MSM mice, whereas only one out of 14 pairs of B6 mice showed very brief aggressive behavior. Among the animals that exhibited aggressive behavior, the duration was longer in MSM $(19.8 \pm 15.2 \mathrm{~s})$ than in B6 mice (0.6 s).

Social interaction behavior in consomic mouse strains

To map the chromosomes that were associated with the social behavior observed in the social interaction test, we analyzed a panel of consomic mouse strains. For the Chr Y strain, we examined only males because females of this strain are genetically identical to B6. No behavioral effect of MSM Chr Y on social behavior was observed (Fig. 2a, b), therefore, we excluded $\mathrm{Chr} \mathrm{Y}$ from the statistical analysis to examine the effect of sex for each chromosome. Two-way ANOVA showed a significant main effect of strain on the duration of social contact $[F(21,442)=$ 15.162, $P<0.001]$. In addition, there was a significant effect of sex $[F(1,442)=5.515, P=0.019]$, and male mice exhibited longer contact than female mice. However, given that we observed no significant interaction between sex and strain, we combined the data for the duration of social contact from male and female mice. Post hoc $t$-tests with Bonferroni correction indicated that consomic strains for Chr 6 (both 6C and 6T) and Chr 17 showed significantly longer contact, whereas strains for Chr 9 and Chr X (both XT and XC) showed shorter contact, as compared to B6 (Fig. 2a). With respect to the frequency of social contact, a significant main effect of strain $[F(21,442)=$ 3.003, $P<0.001)$ and an interaction between strain and sex $[F(21,442)=10.868, P<0.001)$ were observed. In male mice, consomic strains for Chr 2 (both 2C and 2T), Chr 3, Chr 4, Chr 13 and Chr 14 showed a significantly higher frequency of social contact, whereas this was lower in Chr 6C, as compared to strain B6. In contrast, only three strains, Chr 2C, Chr 6C and Chr 14, showed a significant difference from B6 in female mice. A significant difference was observed for sex in the Chr 2T, Chr 3, Chr 4, Chr 7T and Chr 13 strains.

Detailed behavioral analyses were performed on recorded videos of male mice by a well-trained observer. One-way 
Fig. 2 Social contact behavior in consomic mouse strains and B6. a Duration of social contact. Male and female data were combined because there was no strain-sex interaction.

* $P<0.05$ compared to B6. b Frequency of social contact in males and females. * Indicates significant differences with respect to the corresponding sex from B6 $(P<0.05)$. \# Indicates significant sex differences within a strain $(P<0.05)$. The horizontal dotted line shows the mean for B6. MSM was excluded from the ANOVA analysis
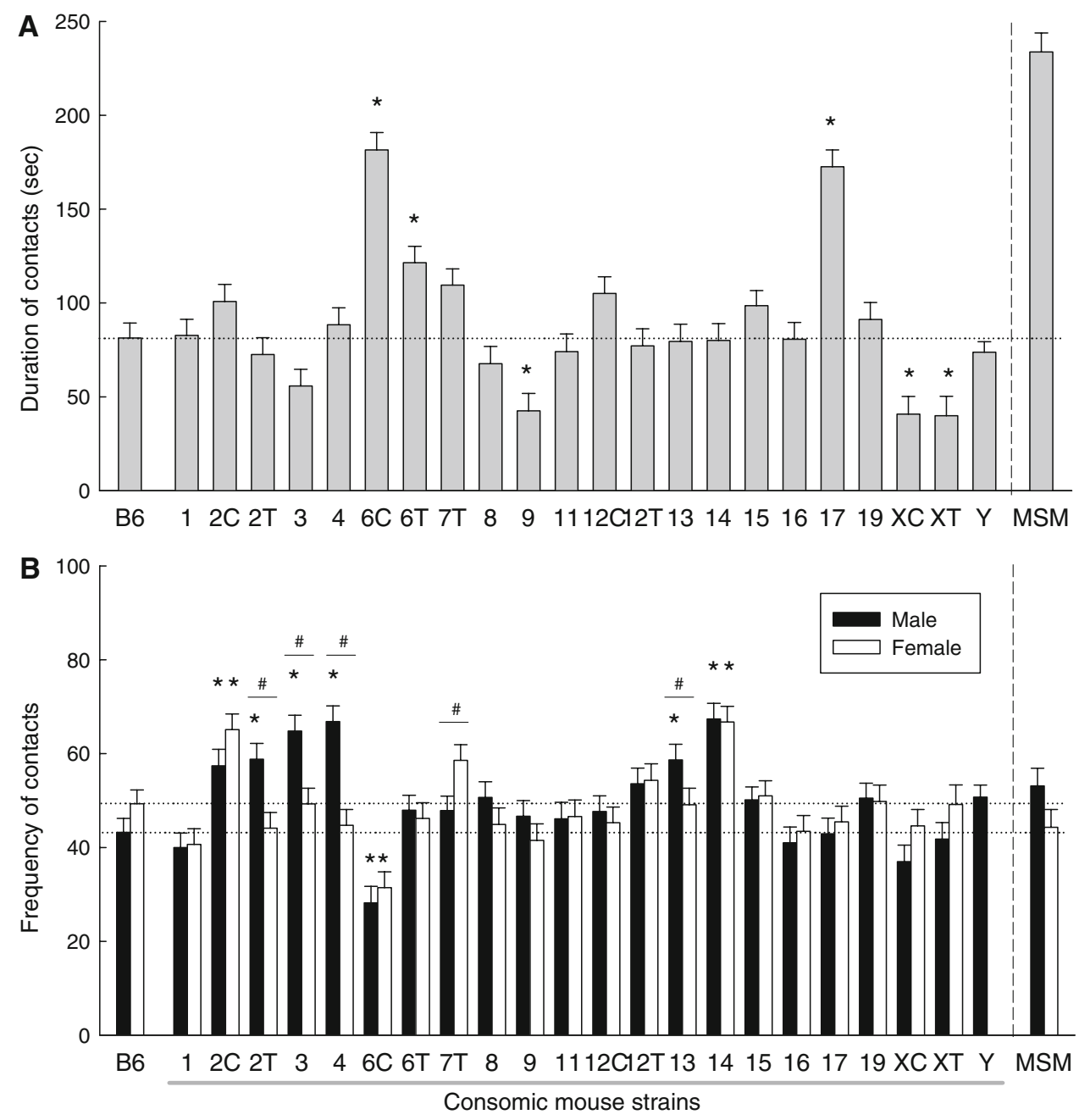

ANOVA indicated a significant main effect of strain for genital grooming $[F(22,237)=8.680]$, social sniffing $[F$ $(22,237)=9.486]$ and following $[F(22,237)=4.691$; for all $P<0.001]$. Post-hoc tests determined that male mice of seven strains, Chr 4, Chr 6C, Chr 8, Chr 9, Chr 12T, Chr 13 and $\mathrm{Chr}$ 17, showed significant changes in some of these types of behavior when compared to B6. Consomic strains for $\mathrm{Chr} 13$ and $\mathrm{Chr} 17$ showed an increase in genital grooming, whereas male mice of the $\mathrm{Chr} 8, \mathrm{Chr} 9$ and $\mathrm{Chr}$ $12 \mathrm{~T}$ strains showed a reduction (Fig. 3a). Male mice of the Chr 4, Chr 6C and Chr 17 strains showed a longer duration of social sniffing, as was observed in MSM mice, when compared to B6 (Fig. 3b). Only one consomic strain, Chr 13, showed a significantly longer duration of following behavior (Fig. 3c). The significant differences among these strains, which were revealed by the post-hoc tests, were confirmed by the $Z$ scores that were estimated using the equation of Belknap(2003) (Supplemental Table). Agonistic behavior was observed in some pairs of male mice from the $\mathrm{Chr}$ 4, Chr 6C, Chr 13, Chr 15 and Chr 17 strains. In particular, in animals that exhibited aggressive behavior,
Chr 4, Chr 13 and Chr 15 showed a long duration of aggressive behavior (Fig. 4).

Correlation between social interaction and anxiety-like behavior

We examined the genetic correlations among types of social interaction behavior using the data from the male mice (Table 2). Duration of social contact had a positive correlation with genital grooming $(r=0.743)$, social sniffing $(r=0.582)$ and following $(r=0.464)$, whereas frequency of social contact did not have any correlation with any of the detailed observations. In addition, positive correlations were observed between aggressive behavior and genital grooming $(r=0.678)$, social sniffing $(r=$ $0.655)$, and following $(r=0.850)$. We also estimated correlation of male and female on duration and frequency of social contact to examine whether the same genetic factors influence both male and female. Pearson's correlation analysis showed positive correlation between male and female on duration of social contact $(r=0.866$, 
Fig. 3 Detailed social interaction behavior in males from the consomic mouse strains and B6. a Genital grooming. b Social sniffing. c Following. The horizontal dotted line shows the mean for B6. Asterisks indicate significant differences as compared to B6 $(P<0.05)$. MSM was excluded from the ANOVA analysis
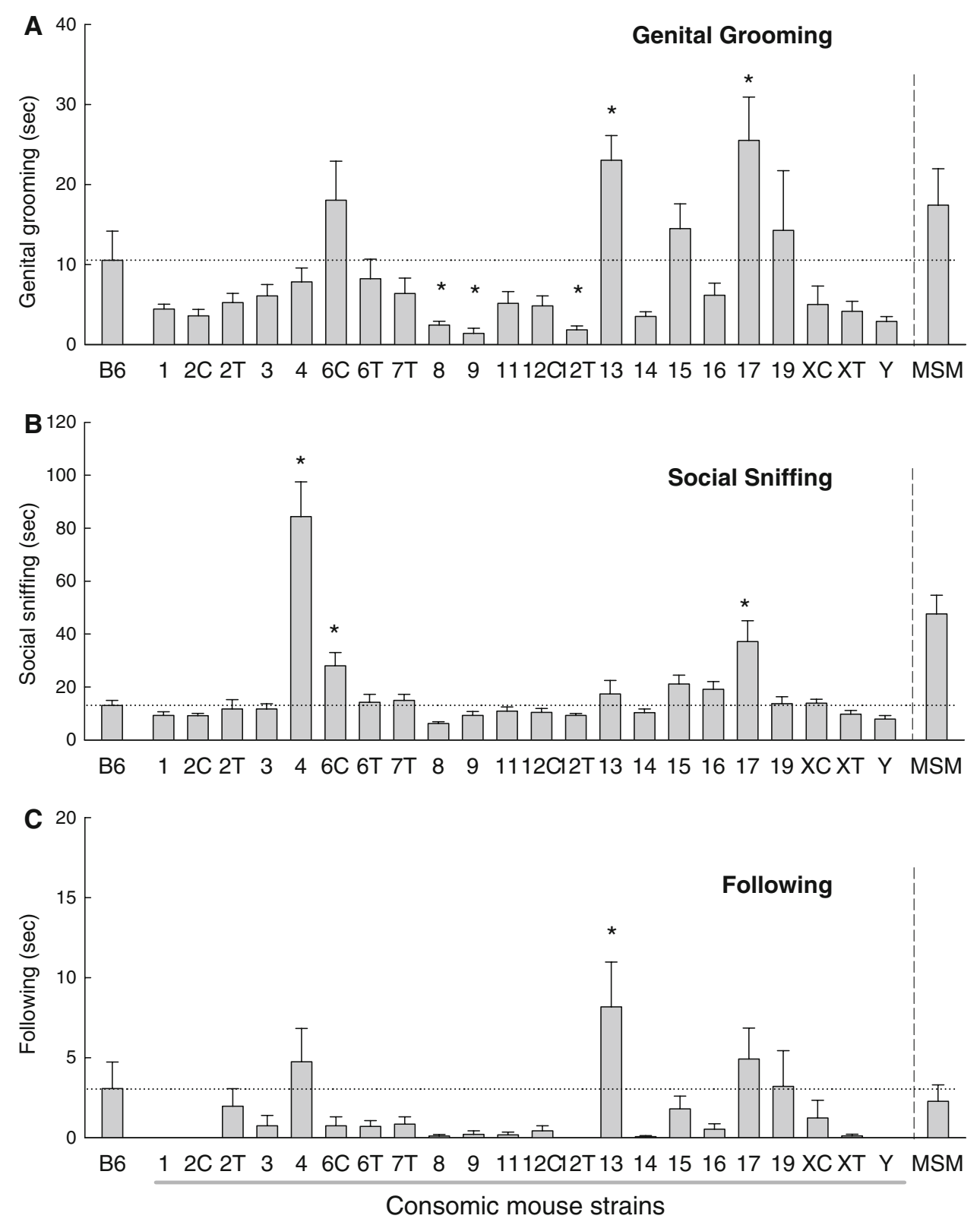

$P<0.001)$ and moderate correlation on the frequency ( $r=0.589, P=0.004)$.

The social interaction test is considered to be a model of anxiety and is sensitive to anxiolytic drugs (File and Hyde 1978; File 1980). Therefore, we examined the genetic correlation of this behavior with other anxiety-like behavior. Previously, we have characterized the consomic strains in three behavioral models of anxiety, the open-field test, elevated plus maze test (EP), and light/dark test, and factor analysis identified five factors that underlie anxiety-like behavior (locomotor activity, thigmotaxis, EP open-arm exploration, risk assessment, and autonomic emotionality; Takahashi et al. 2008). Here, we examined the genetic correlation between these five factors and social interaction behavior in males (Table 3). Duration of social contact had a negative correlation with general locomotor activity $(r=-0.578)$, and a positive correlation with thigmotaxis $(r=0.417)$ and autonomic emotionality $(r=0.727)$. In contrast, frequency of contact showed a positive correlation with general locomotor activity $(r=0.647)$ and a negative correlation with autonomic emotionality $(r=-0.661)$. For detailed behavior, genital grooming showed a positive correlation with autonomic emotionality $(r=0.503)$. No strong correlations were found between anxiety-like behavior and social sniffing, following, or aggressive behavior. 


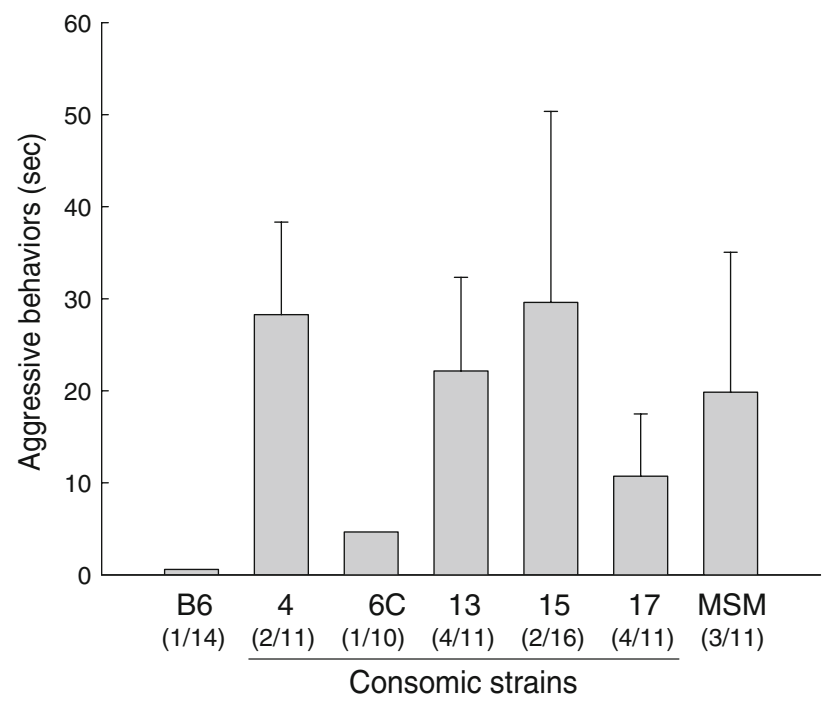

Fig. 4 Aggressive behavior in consomic strains and parental B6 and MSM. Figure shows the average duration of aggressive behavior (both attack and aggressive chasing) in animals that exhibited aggressive behavior. The numbers in parentheses show the number of pairs in each strain that showed aggressive behavior. Aggressive behavior was observed only in the five consomic strains shown in this figure

\section{Discussion}

The wild-derived strain MSM (M. m. molossinus) showed a significantly longer duration of social contact than B6 mice for both sexes. Detailed behavioral analysis revealed that the long duration of contact in MSM resulted from longer periods of social sniffing as compared to B6 mice. In addition, we noticed that the MSM mice tended to stay together in the same corner without performing any active social behavior, such as genital grooming or social sniffing. Therefore, our results suggest that MSM mice have a high level of social affiliation in the novel open-field situation. The social interaction test has been used as a valid measurement of anxiety (Crawley 2007; Overstreet 2007) because this behavior changes with intensity of stress, and pharmacological manipulation can modulate the outcome. It has been shown that conditions of high illumination or unfamiliarity with the arena suppress social interaction behavior (File and Hyde 1978), whereas anxiolytics such as benzodiazepines increase social interaction (File 1980). Therefore, the longer duration of social interaction in MSM mice may be associated with low levels of anxiety. However, when MSM mice were tested alone in the open-field, they showed low locomotor activity, high levels of freezing and self-grooming behavior, and they took a long time to habituate to novelty. In contrast, B6 mice exhibited the highest activity among 12 strains and habituated quickly to the novel open-field (Takahashi et al. 2006). MSM mice also showed higher levels of anxiety-like behavior than B6 in the light/dark test (Takahashi et al. 2008). We reported previously the results of systematic analyses of anxiety-like behavior in a B6-ChrN ${ }^{\mathrm{MSM}}$ consomic panel (Takahashi et al. 2008). In the present study, we examined further the relationship between social interaction and other anxietylike behavior in the consomic panel. Genetic correlation analysis showed that the duration of social contact had a negative correlation with general locomotor activity and a positive correlation with thigmotaxis in an open-field, and autonomic emotionality, which is characterized by defecation (Table 3). Again, as observed in MSM mice, we found different associations between different types of social contact and other anxiety-like behavior in consomic strains. Genital grooming showed a positive correlation with open-arm exploration in the elevated plus maze; however, the correlation was modest. This inconsistent relationship between the results of the social interaction test and other anxiety-like behavior has been observed in some knockout mice, in which social interaction behavior was changed but other anxiety-like behavior was unaltered, compared to that in the wild-type mice (Sano et al. 2009, O'Tuathaigh et al. 2008). These results suggest that the social interaction test measures a different aspect of anxiety from the anxiety-like behavior that is measured by the open-field, elevated plus-maze and light/dark tests.

Table 2 Genetic correlations in social interaction behavior

\begin{tabular}{|c|c|c|c|c|c|c|}
\hline & \multicolumn{6}{|c|}{ Genetic correlations } \\
\hline & $\begin{array}{l}\text { Contact } \\
\text { duration }\end{array}$ & $\begin{array}{l}\text { Contact } \\
\text { frequency }\end{array}$ & $\begin{array}{l}\text { Genital } \\
\text { grooming }\end{array}$ & $\begin{array}{l}\text { Social } \\
\text { sniffing }\end{array}$ & Following & $\begin{array}{l}\text { Aggressive } \\
\text { behavior }\end{array}$ \\
\hline Contact duration & & $-0.189(P=0.388)$ & $0.743(P<0.001 * *)$ & $0.582\left(P=0.004^{* *}\right)$ & $0.464\left(P=0.026^{*}\right)$ & $0.373(P=0.080)$ \\
\hline Contact frequency & & & $-0.099(P=0.652)$ & $0.064(P=0.771)$ & $0.217(P=0.320)$ & $0.278(P=0.198)$ \\
\hline Genital grooming & & & & $0.585\left(P=0.003^{* *}\right)$ & $0.803(P<0.001 * *)$ & $0.678(P<0.001 * *)$ \\
\hline Social sniffing & & & & & $0.593\left(P=0.003^{* *}\right)$ & $0.655(P<0.001 * *)$ \\
\hline Following & & & & & & $0.850(P<0.001 * *)$ \\
\hline
\end{tabular}

Genetic correlations of $r>0.60$ are in bold, and $r<0.30$ are in italics. $P$ values are provided in parentheses

** $P<0.01, * P<0.05$ 


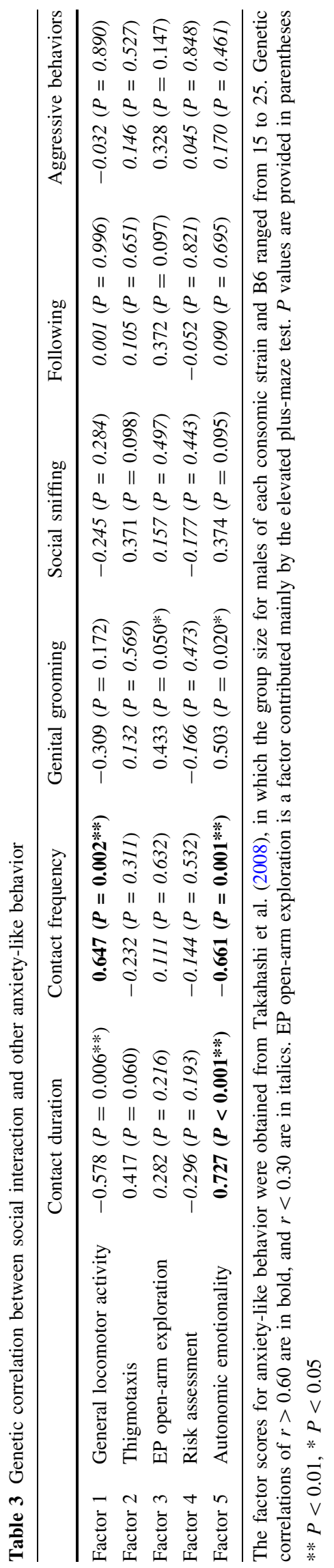

Multivariate analysis in rats showed that the factors that affect social interaction behavior are distinct from those that affect the behavior measured in the open-field or plusmaze tests (Berton et al. 1997). It is also possible that MSM mice, as well as some consomic strains, have a strong motivation to exhibit social contact (in other words, social affiliation) that can overcome the behavioral inhibition that is induced by novelty. Accordingly, we expect that the genetic mapping of social interaction behavior will identify QTLs for aspects of anxiety other than those measured in non-social situations, or QTLs that are related to social affiliation.

By using a panel of B6-ChrN ${ }^{\mathrm{MSM}}$ consomic strains, we identified chromosomes that were associated with social interaction behavior. To the best of our knowledge, this is the first report that describes genetic loci for social interaction behavior, other than aggressive behavior (Brodkin et al. 2002; Roubertoux et al. 2005). The effect of Chr 6 and $\mathrm{Chr} 17$ on the duration of social contact stood out. These strains showed twofold longer social contact than the parental B6 strain, and exhibited longer durations of genital grooming and social sniffing. For $\mathrm{Chr} 6$, we examined two subconsomic strains (6C and 6T) in this study: in $6 \mathrm{C}$ a prominent effect on the duration of social contact was observed but in $6 \mathrm{~T}$ there was only a moderate effect. This indicates that there are at least 2 QTLs for this behavior on Chr 6. Chr 4 had a very strong effect on social sniffing, and in the Chr 13 strain, the amount of genital grooming and following was increased as compared to B6. Although the pattern of effects of $\mathrm{Chr} 4, \mathrm{Chr} 6, \mathrm{Chr} 13$ and Chr 17 on social interaction was different, these four chromosomes contain QTLs that facilitate social behavior in MSM mice. In contrast, social interaction behavior was decreased in the $\mathrm{Chr} \mathrm{8,} \mathrm{Chr} \mathrm{9,} \mathrm{Chr} 12$ and Chr X strains. Further fine mapping of the QTLs on these chromosomes is required to identify the genes that are related to social interaction behavior. This will allow us to describe more precisely the relationship between social interaction and anxiety-like behavior. In addition, the results of this study may give us insights into the genetic factors that underlie aberrant social behavior (e.g., autism). In humans, genome-wide linkage analysis has identified several QTLs associated with autism. Meta-analysis of nine genome scans on autism identified a consistent QTL on 7q22-q32 (Trikalinos et al. 2006). This chromosomal location corresponds to $\mathrm{Chr} 5$ and the centromeric portion of $\mathrm{Chr} 6$ in mice. In our study, social interaction behavior was enhanced strongly in the $\mathrm{Chr} 6 \mathrm{C}$ subconsomic strain (we did not test the consomic Chr 5 strain), and it is possible that polymorphisms in the same gene may cause autism in humans, and affect social interaction behavior in mice. In addition, one of the best-known genes associated with autism, Mecp2 (Amir et al. 1999; Nagarajan et al. 2006; 
Thatcher et al. 2005), is located on Chr X in both humans and mice, and we found that the $\mathrm{Chr} \mathrm{X}$ consomic strain showed reduced social contact in the novel open-field. However, more detailed genetic and behavioral analysis will be required to examine the relationship with autism.

Frequency of social contact shows very different characteristics to other types of social interaction behavior. Correlation analysis revealed that frequency of contact showed almost no correlation with other social behavior (Table 2), but did show a strong genetic correlation with general locomotor activity in novel test situations (Table 3). Our video tracking system detected social contact when the centers of the two mice came within a distance of $12 \mathrm{~cm}$ of each other, and we suspect that highactivity animals have a greater probability of coming close to each other in the absence of any active social behavior. We found that animals of both sexes from the Chr $2 \mathrm{C}$ and Chr 14 strains, males from the Chr 2T and Chr 3 strains, and females from the $\mathrm{Chr} 7 \mathrm{~T}$ strain showed increased frequency of social contact as compared to B6 mice, but did not differ in the other types of social interaction behavior. Most of these strains, except for the Chr 2 consomic strain, exhibited high general locomotor activity in our previous study (Takahashi et al. 2008). For the Chr 2 mice, it is likely that social context enhanced their locomotor activity.

A prominent sex-strain interaction was observed with respect to frequency of social contact. Correlation analysis between male and female on frequency of social contact showed only modest correlation $(r=0.589)$. We expect that chromosomes that result in a significant sex difference contain QTLs that are responsible for a sex-specific effect. Our results show that QTLs on Chr 2T, Chr 3, Chr 4 and Chr 13 have a male-specific effect, and the QTL on Chr 7T has a female-specific effect. Sex-specific QTLs have been reported for anxiety-like behavior (Ramos et al. 1999; Takahashi et al. 2008), fear conditioning (Ponder et al. 2007), pain (Devor et al. 2007), and depression-like behavior (Solberg et al. 2004). To understand the effects of QTLs in more detail, it is useful to examine differences between the sexes. For Chr 2, our results suggest that there are at least two QTLs: one in the centromeric portion of the chromosome that affects the frequency of social contact in both sexes, and another in the telomeric portion that affects only male mice. However, there is a methodological limitation of our study with respect to the sex-genotype interaction: we did not follow the estrus cycle in the female mice. The behavior of female mice and rats, including anxiety-like behaviors, changes in a manner that depends on the stage of the estrus cycle (Guttman et al. 1975, Mora et al. 1996). To investigate the sex-specific effects of the loci in more detail, it will be necessary to consider the estrus cycle of the female mice. In addition, we did not make detailed behavioral observations of the females. It is possible that females of some consomic strains may show different behavioral patterns that were not detected by automatic video analysis.

We observed that, after weaning, some male MSM mice attacked and injured, and sometimes killed, littermates of the same sex. Therefore, we expect that the consomic strains established from MSM will be a useful tool to examine the genetic basis of aggression. Although it was not statistically significant, aggressive behavior was observed in some pairs from the consomic strains for $\mathrm{Chr} 4, \mathrm{Chr}$ 13, Chr 15 and $\mathrm{Chr}$ 17. Increased aggression of a $\mathrm{Chr} 4$ consomic strain has been reported also for an A/J-B6 consomic panel (Singer et al. 2005). In contrast, several studies have shown the importance of $\mathrm{Chr} \mathrm{Y}$ in inter-male aggression in mice and rats (Guillot et al. 1995; Maxson et al. 1989; Roubertoux et al. 1994; Toot et al. 2004). In addition, QTLs for mouse aggressive behavior have been reported on $\mathrm{Chr} 8, \mathrm{Chr} 9, \mathrm{Chr}$ 11, Chr 12 and Chr X (Brodkin et al. 2002; Roubertoux et al. 2005). However, our B6-MSM consomic strains for Chr 8, Chr 9, Chr 11, Chr 12 and the sex chromosomes did not exhibit aggressive behavior in the social interaction test, but rather showed reduced social interaction behavior. These inconsistencies may have resulted from differences in the mouse strains used or the context of the behavioral tests. In addition, the occurrence of aggressive behavior in the novel open-field was low because novelty inhibits social behavior; hence, we could not detect statistical significance. To obtain more reliable results, it will be useful to perform home-cage resident-intruder tests to investigate aggressive behavior in these consomic strains.

There are several methodological issues to be considered in this study. First, we examined social interaction behavior only in homogeneous sets, and not with a standard opponent. Fuller and Hahn (1976) proposed that the genetics of social behavior can be examined by three types of experimental design: with homogeneous sets, with a standard opponent, and with a panel of opponents. The social behaviors of an animal can be affected by the stimulus animal used (Selmanoff et al. 1976; Maxson and Canastar 2003; Ogawa et al. 2004; Roubertoux et al. 2005). The advantage of using inbred strains rather than F2 heterogeneous individuals is that it allows us to examine both homogeneous sets (interaction between the same genotype) and standard opponent/opponents (interaction between different genotypes). Further studies to investigate the effect of the opponent will be required. Another issue in this study was that all behavioral tests were performed during the light period. Mice are nocturnal animals and they have a lower body temperature and lower activity in the open-field during the light phase than during the dark phase (Connolly and Lynch 1981). This factor should be considered in the interpretation of the results. 
This study revealed the genome-wide profiles of genetic factors that are related to social interaction behavior, perhaps for the first time. Additional genetic studies will help to further our understanding of the genetic mechanisms by which social behavior is regulated, including aggressive and social-context-induced anxiety-like behavior.

Acknowledgments This study was supported by the Japan Society for the Promotion of Science; a Grant-in-Aid for Scientific Research (KAKENHI) on the Priority Area "Comparative Genomics" from the Ministry of Education, Culture, Sports, Science and Technology, NIG Cooperative Research Program (2008-A34) and the Research Organization of Information and Systems, Transdisciplinary Research Integration Center.

Open Access This article is distributed under the terms of the Creative Commons Attribution Noncommercial License which permits any noncommercial use, distribution, and reproduction in any medium, provided the original author(s) and source are credited.

\section{References}

Amir RE, Van den Veyver IB, Wan M, Tran CQ, Francke U, Zoghbi HY (1999) Rett syndrome is caused by mutations in X-linked MECP2, encoding methyl-CpG-binding protein 2. Nat Genet 23:185-188

Belknap JK (2003) Chromosome substitution strains: some quantitative considerations for genome scans and fine mapping. Mamm Genome 14:723-732

Berton O, Ramos A, Chaouloff F, Mormede P (1997) Behavioral reactivity to social and nonsocial stimulations: a multivariate analysis of six inbred rat strains. Behav Genet 27:155-166

Blizard DA, Bailey DW (1979) Genetic correlation between openfield activity and defecation: analysis with the CXB recombinant-inbred strains. Behav Genet 9:349-357

Brodkin ES, Goforth SA, Keene AH, Fossella JA, Silver LM (2002) Identification of quantitative trait loci that affect aggressive behavior in mice. J Neurosci 22:1165-1170

Brunner HG, Nelen MR, van Zandvoort P, Abeling NG, van Gennip AH, Wolters EC, Kuiper MA, Ropers HH, van Oost BA (1993a) $\mathrm{X}$-linked borderline mental retardation with prominent behavioral disturbance: phenotype, genetic localization, and evidence for disturbed monoamine metabolism. Am J Hum Genet 52:1032-1039

Brunner HG, Nelen M, Breakefield XO, Ropers HH, van Oost BA (1993b) Abnormal behavior associated with a point mutation in the structural gene for monoamine oxidase A. Science 262: $578-580$

Choleris E, Kavaliers M, Pfaff DW (2004) Functional genomics of social recognition. J Neuroendocrinol 16:383-389

Connolly MS, Lynch CB (1981) Circadian variation of strain differences in body temperature and activity in mice. Physiol Behav 27:1045-1049

Crawley JN (2007) What's wrong with my mouse?, 2nd edn. Wiley, Hoboken

de Angelis L, File SE (1979) Acute and chronic effects of three benzodiazepines in the social interaction anxiety test in mice. Psychopharmacology 64:127-129

Devor M, Gilad A, Arbilly M, Nissenbaum J, Yakir B, Raber P, Minert A, Pisanté A, Darvasi A (2007) Sex-specific variability and a 'cage effect' independently mask a neuropathic pain quantitative trait locus detected in a whole genome scan. Eur J Neurosci 26:681-688

Donaldson ZR, Young LJ (2008) Oxytocin, vasopressin, and the neurogenetics of sociality. Science 322:900-904

File SE (1980) The use of social interaction as a method for detecting anxiolytic activity of chlordiazepoxide-like drugs. J Neurosci Methods 2:219-238

File SE, Hyde JR (1978) Can social interaction be used to measure anxiety? Br J Pharmacol 62:19-24

Flint J (2003) Analysis of quantitative trait loci that influence animal behavior. J Neurobiol 54:46-77

Fuller JL, Hahn ME (1976) Issues in the genetics of social behavior. Behav Genet 6:391-406

Gammie SC (2005) Current models and future directions for understanding the neural circuitries of maternal behaviors in rodents. Behav Cogn Neurosci Rev 4:119-135

Guillot P-V, Carlier M, Maxson SC, Roubertoux PL (1995) Intermale aggression tested in two procedures, using four inbred strains of mice and their reciprocal congenics: Y chromosomal implications. Behav Genet 25:357-360

Guttman R, Lieblich I, Gross R (1975) Behavioral correlates of estrous cycle stages in laboratory mice. Behav Biol. 13:127-132

Koide T, Moriwaki K, Ikeda K, Niki H, Shiroishi T (2000) Multiphenotype behavioral characterization of inbred strains derived from wild stocks of Mus musculus. Mamm Genome 11:664-670

Maxson SC, Canastar A (2003) Conceptual and methodological issues in the genetics of mouse agonistic behavior. Horm Behav 44:258-262

Maxson SC, Didier-Erickson A, Ogawa S (1989) The Y chromosome, social signals, and offense in mice. Behav Neural Biol 52:251259

Miczek KA, Maxson SC, Fish EW, Faccidomo S (2001) Aggressive behavioral phenotypes in mice. Behav Brain Res 125:167-181

Mora S, Dussaubat N, Díaz-Véliz G (1996) Effects of the estrous cycle and ovarian hormones on behavioral indices of anxiety in female rats. Psychoneuroendocrinology 21:609-620

Moriwaki K, Miyashita N, Mita A, Gotoh H, Tsuchiya K, Kato H, Mekada K, Noro C, Oota S, Yoshiki A, Obata Y, Yonekawa H, Shiroishi T (2009) Unique inbred strain MSM/Ms established from the Japanese wild mouse. Exp Anim 58:123-134

Nadeau JH, Singer JB, Matin A, Lander ES (2000) Analysing complex genetic traits with chromosome substitution strains. Nature Genet 24:221-225

Nagarajan RP, Hogart AR, Gwye Y, Martin MR, LaSalle JM (2006) Reduced MeCP2 expression is frequent in autism frontal cortex and correlates with aberrant MECP2 promoter methylation. Epigenetics 1:e1-e11

Nelson RJ, Chiavegatto S (2001) Molecular basis of aggression. Trends Neurosci 24:713-719

O'Tuathaigh CM, O'Connor AM, O’Sullivan GJ, Lai D, Harvey R, Croke DT, Waddington JL (2008) Disruption to social dyadic interactions but not emotional/anxiety-related behaviour in mice with heterozygous 'knockout' of the schizophrenia risk gene neuregulin-1. Prog Neuropsychopharmacol Biol Psychiatry 32(2):462-466

Ogawa S, Choleris E, Pfaff DW (2004) Genetic influences on aggressive behaviors and arousability in animals. Ann NY Acad Scie 1036:257-266

Overstreet DH (2007) The open field test for two. J Psychopharmacol 21:140

Pfaff D, Frohlich J, Morgan M (2002) Hormonal and genetic influences on arousal-sexual and otherwise. Trends Neurosci 25:45-50

Ponder CA, Munoz M, Gilliam TC, Palmer AA (2007) Genetic architecture of fear conditioning in chromosome substitution 
strains: relationship to measures of innate (unlearned) anxietylike behavior. Mamm Genome 18:221-228

Ramos A, Moisan MP, Chaouloff F, Mormede C, Mormede P (1999) Identification of female-specific QTLs affecting an emotionalityrelated behavior in rats. Mol Psychiatry 4:453-462

Roubertoux PL, Carlier M, Degrelle H, Haas-Dupertuis MC, Phillips J, Moutier R (1994) Co-segregation of intermale aggression with the pseudoautosomal region of the $\mathrm{Y}$ chromosome in mice. Genetics 136:225-230

Roubertoux PL, Guillot P-V, Mortaud S, Pratte M, Jamon M, CohenSalmon C, Tordjman S (2005) Attack behaviors in mice: from factorial structure to quantitative trait loci mapping. Eur $\mathrm{J}$ Pharmacol 526:172-185

Sano Y, Ornthanalai VG, Yamada K, Homma C, Suzuki H, Suzuki T, Murphy NP, Itohara S (2009) X11-like protein deficiency is associated with impaired conflict resolution in mice. J Neurosci 29:5884-5896

Selmanoff MK, Maxson SC, Ginsburg BE (1976) Chromosomal determinants of intermale aggressive behavior in inbred mice. Behav Genet 6:53-69

Singer JB, Hill AE, Nadeau JH, Lander ES (2005) Mapping quantitative trait loci for anxiety in chromosome substitution strains of mice. Genetics 169:855-862

Solberg LC, Baum AE, Ahmadiyeh N, Shimomura K, Li R, Turek FW, Churchill GA, Takahashi JS, Redei EE (2004) Sex- and lineage-specific inheritance of depression-like behavior in the rat. Mamm Genome 15:648-662

Takada T, Mita A, Maeno A, Shitara H, Kikkawa Y, Moriwaki K, Yonekawa H, Shiroishi T (2008) Mouse inter-subspecific consomic strains for genetic dissection of quantitative complex traits. Genome Res 18:500-508

Takahashi A, Kato K, Makino J, Shiroishi T, Koide T (2006) Multivariate analysis of temporal descriptions of open-field behavior in wild-derived mouse strains. Behav Genet 36:763-774

Takahashi A, Nishi A, Ishii A, Shiroishi T, Koide T (2008) Systematic analysis of emotionality in consomic mouse strains established from C57BL/6 J and wild-derived MSM/Ms. Genes Brain Behav 7:849-858

Thatcher KN, Peddada S, Yasui DH, Lasalle JM (2005) Homologous pairing of 15q11-13 imprinted domains in brain is developmentally regulated but deficient in Rett and autism samples. Hum Mol Genet 14:785-797

Toot J, Dunphy G, Turner M, Ely D (2004) The SHR Y-chromosome increases testosterone and aggression, but decreases serotonin as compared to the WKY Y-chromosome in the rat model. Behav Genet 34:515-524

Trikalinos TA, Karvouni A, Zintzaras E, Ylisaukko-oja T, Peltonen L, Järvelä I, Ioannidis JP (2006) A heterogeneity-based genome search meta-analysis for autism-spectrum disorders. Mol Psychiatry $11: 29-36$ 